The Astrophysical Journal, 192: 379-382, 1974 September 1

(C) 1974. The American Astronomical Society. All rights reserved. Printed in U.S.A.

\title{
OPTICAL INTERSTELLAR LINES IN DARK CLOUDS. II. K I AND ULTRAVIOLET SODIUM LINES
}

\author{
JUDITH G. COHEN* \\ Kitt Peak National Observatory† \\ Received 1974 February 6; revised 1974 March 25
}

\begin{abstract}
New observations of the $\mathrm{K} \mathrm{I}$ and ultraviolet $\mathrm{Na}$ I interstellar lines are presented for some stars embedded in dense clouds and for a few supergiants. The new observations of weak lines, which are essentially unaffected by the adopted velocity distribution for the interstellar material, support the conclusions previously obtained from the interstellar D-lines of $\mathrm{Na}$ I and $\mathrm{H}$ - and $\mathrm{K}$-lines of $\mathrm{Ca}$ II. The new data, when combined with a discussion of the ionization equilibrium, indicate no or moderate depletions at the edges of clouds, but some depletions in the central regions.
\end{abstract}

Subject headings: abundances, nebular — interstellar matter — nebulae

\section{INTRODUCTION}

In Cohen (1973, hereafter referred to as Paper I), I compared the behavior of the optical interstellar lines in a group of stars behind dense clouds with those in some highly reddened supergiants. I concluded that the atomic forms of $\mathrm{Ca}$, and to a lesser extent $\mathrm{Na}$, are missing in the central regions of dark clouds. One of the principal difficulties in the comparison of the column densities deduced from my observations of equivalent widths $\left(W_{\lambda}\right)$ is that the interstellar lines of the supergiants, which are more distant than the cloud stars, are affected by galactic rotation. Through new observations of the ultraviolet $\mathrm{Na}$ I doublet for some of the stars in Paper I, we show that the procedures used to analyze the earlier data do not lead to gross errors, and that the effects described in the earlier work are also seen in the ultraviolet $\mathrm{Na} I$ lines. Hence, the conclusions of Paper I are strengthened by the new observations. A brief discussion of the behavior of the $\mathrm{K}$ I line $(\lambda 7699)$ is also given. I also rediscuss the ionization equilibrium calculations of Paper I using the new observations.

\section{THE Na I OBSERVATIONS}

In Paper I, I assumed that the line of sight to a supergiant intersects sufficiently many clouds randomly distributed in velocity within the range permitted by galactic rotation that one may use the standard curve of growth for a single cloud with a Gaussian velocity distribution whose velocity dispersion is $b_{\text {eff }}=\left(b_{e, 0}^{2}+b_{\text {gal rot }}^{2}\right)^{0.5}$ in the notation of Paper I. I also assumed that the velocity distribution in the dark clouds can be adequately represented by a Gaussian. To verify that this procedure is adequate, one must compare the column density derived from a very weak line, where the velocity structure does not

* Guest observer at Hale Observatories, Pasadena, California.

$\dagger$ Operated by the Association of Universities for Research in Astronomy, Inc., under contract with the National Science Foundation. affect $W_{\lambda}$, with that derived from a strong feature of the same ion whose equivalent width is dependent on the velocity distribution. The most favorable case for such a comparison is $\mathrm{Na} \mathrm{I}$, as the yellow D-lines are strongly saturated (more so than $\mathrm{H}$ - and $\mathrm{K}$-lines of Ca II), while the ultraviolet doublet at $3303 \AA$ is very weak. The observational difficulties are formidable. The atmospheric transmission is low; the stars of interest, which are faint in the visual, are reddened with $E_{B-V}$ of up to $1.2 \mathrm{mag}$, so that the ultraviolet magnitudes are depressing. However, persistence with the 2.5-m Hooker telescope of the Mount Wilson Observatory has yielded six new measurements of the ultraviolet $\mathrm{Na}$ I doublet for the stars of Paper I. The three measurements of the Perseus stars were obtained from $4.5 \AA \mathrm{mm}^{-1}$ spectra, the others with $9 \AA \mathrm{mm}^{-1}$ spectra (all baked IIa-O emulsion). We add the five previously reported measurements to the new observations and display the data in table 1 . The color

TABLE 1

Ultraviolet Na i ObServations

\begin{tabular}{|c|c|c|c|c|c|c|}
\hline \multirow[b]{2}{*}{ STAR } & \multirow[b]{2}{*}{$E_{B-V}$} & \multicolumn{2}{|c|}{$W_{\lambda}(\mathrm{m} \AA)$} & \multirow[b]{2}{*}{$N_{U V}$} & \multirow[b]{2}{*}{$N_{D}$} & \multirow[b]{2}{*}{ SoURCE } \\
\hline & & 3302.4 & 3303.0 & & & \\
\hline \multicolumn{7}{|l|}{ Cloud stars: } \\
\hline$\rho$ Oph AB. & 0.47 & 30 & 15 & 13.5 & 12.4 & JC \\
\hline $\begin{array}{l}\mathrm{Per} \\
\mathrm{Per}\end{array}$ & $\begin{array}{l}0.38 \\
0.33\end{array}$ & $\begin{array}{l}30 \\
30\end{array}$ & $\begin{array}{l}20^{*} \\
15\end{array}$ & $\begin{array}{l}13.5 \\
13.5\end{array}$ & 12.2 & $\begin{array}{l}\mathrm{JC} \\
\mathrm{JC}\end{array}$ \\
\hline o Per & 0.32 & 50 & 30 & 13.9 & 12.5 & $\mathrm{JC}$ \\
\hline$\zeta \mathrm{Oph}$. & 0.32 & 29 & 22 & 13.7 & 12.5 & $\mathrm{H}$ \\
\hline \multicolumn{7}{|c|}{ Thin interstellar medium stars: } \\
\hline HD 192660 . & 0.88 & 80 & 60 & 14.1 & 13.3 & $\mathrm{JC}$ \\
\hline HD $34078 \ldots$. & 0.78 & 85 & & 14.0 & 13.1 & $\mathrm{~S}$ \\
\hline$\psi^{2}$ Ori $\ldots .$. & 0.54 & 75 & 37 & 13.9 & 13.1 & SF \\
\hline HD 54662 & 0.35 & 54 & & 13.7 & 13.4 & $\mathbf{S}$ \\
\hline HD 170580A. & 0.34 & 50 & 40 & 14.0 & 12.8 & $\mathrm{JC}$ \\
\hline HD 188209 & 0.25 & 104 & & 14.1 & 12.8 & \\
\hline
\end{tabular}

* Doublet ratio assumed to be 2 .

SourCES: H, Herbig 1968; S, Scholz 1972; SF, Spitzer and Field 1955. 

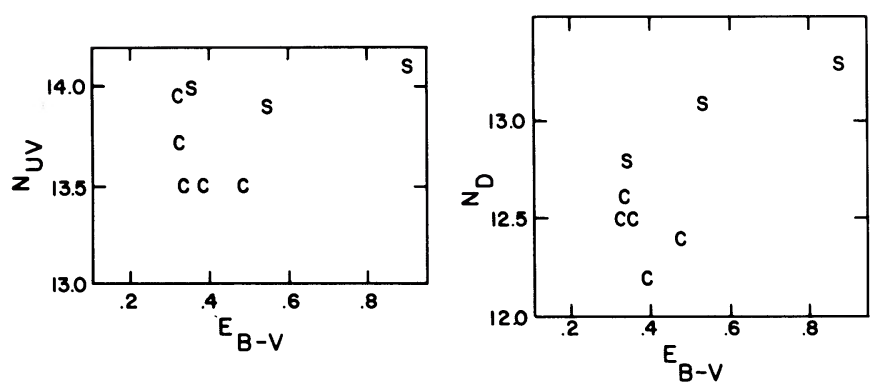

FIG. 1.-Logarithm of the column density of $\mathrm{Na}$ I as deduced from the ultraviolet and yellow doublets plotted as a function of color excess. The symbol $\mathbf{S}$ denotes supergiants; $\mathrm{C}$, cloud stars.

excess is also listed, where the $B, V$ colors are from Blanco et al. (1970) and the calibration of $B-V$ with spectral type is that of Schild, Peterson, and Oke (1971). One must recall that this is a difficult measurement, and the uncertainty in $W_{\lambda}$ is larger than in Paper I, especially for the most reddened stars, perhaps \pm 20 percent in $W_{\lambda}$. The column density of $\mathrm{Na}$ I $\left(N_{\mathrm{UV}}\right)$ is derived from the measurements of $W_{\lambda}$ $(3303 \AA$ ) using the $f$-value of Morton and HaydenSmith (1973) and the curve of growth given by Münch (1968) for a Gaussian velocity distribution. The averaged results for the supergiants and the cloud stars are unchanged if we replace $W_{\lambda}(\lambda 3303.0)$ with $W_{\lambda}(\lambda \lambda 3302.4+3303.0) / 1.5$ and assume the doublet ratio is 2 in all cases. The values for $N_{\mathrm{D}}{ }^{1}$ (the $\mathrm{Na}$ I column density deduced from the D-lines using the procedure of Paper I) were taken from Paper I. For stars not included in Paper I, $N_{\mathrm{D}}$ was computed from the D-line equivalent widths measured by the sources listed in table 1 .

\section{THE ULTRAVIOLET Na I RESULTS}

The major result is that $\left(N_{\mathrm{UV}}-N_{\mathrm{D}}\right)$ averaged over the cloud stars is 1.2 , whereas the same quantity averaged over the thin, normal interstellar medium is 0.9 . The average of the supergiants, excluding the three O stars from Scholz (1972), is also 0.9. Since we believe the column densities deduced from the ultraviolet doublet to be unaffected by errors in velocity distributions, we conclude that there is no systematic error greater than a factor of 2 in the derivation of $N_{\mathrm{D}}$ for the cloud stars compared with the supergiants. Since the $\mathrm{Ca}$ II lines are even less saturated, we may expect any such systematic error to be smaller.

In figure $1 a$, we plot $N_{\mathrm{UV}}$ versus $E_{B-V}$, while in figure $1 b$, we plot $N_{\mathrm{D}}$ versus $E_{B-v}$. The trend of less $\mathrm{Na}$ I at a given color excess for the cloud stars as compared with the supergiants is maintained, although it is perhaps not quite as large as that determined from the $\mathrm{D}$-lines. The $\mathrm{O}$ stars are omitted from this plot due to probable differences in ionization caused by the extensive $\mathrm{H}$ in region.

In figure 2 , we plot $\left(N_{\mathrm{UV}}-N_{\mathrm{D}}\right)$ as a function of the doublet ratio of the D-lines. The correlation is good, implying that the difference between $\left(N_{\mathrm{UV}}-N_{\mathrm{D}}\right)$

${ }^{1} N_{\mathrm{D}}$ and $N_{\mathrm{Uv}}$ are the logarithms of the indicated column densities. averaged over the supergiants and the cloud stars is due to the smaller doublet ratios (closer to unity) prevailing among the more saturated and larger values of $W_{\lambda}$ found in the supergiants.

Figure 2 shows a mean difference, $N_{\mathrm{Uv}}-N_{\mathrm{D}}$, of approximately 1.2 for the least saturated D-lines. This is very puzzling, as the $f$-values for both doublets are thought to be well determined. The calculations of Nachman and Hobbs (1973) suggest that the behavior displayed in figure 2 can be explained by a variation in the fraction of matter in the form of cold clouds from the cloud stars to the supergiants combined perhaps with a change in the velocity separation of the components. Some support for this explanation is that one would naturally expect the cloud stars to have a larger fraction of material along the line of sight in the form of cold clouds than the supergiants, which is in the direction required to produce larger values of $N_{\mathrm{UV}}-$ $N_{\mathrm{D}}$ for the cloud stars as observed. However, if in future observations of stars with doublet ratios closer to $2, N_{\mathrm{UV}}-N_{\mathrm{D}}$ continues to be much greater than 0.0 , then another explanation must be sought for the trends of figure 2 .

\section{THE INTERSTELLAR K I OBSERVATIONS}

Observations of the stronger line of the $\mathrm{K}$ I doublet at $7699 \AA$ (the weaker feature is masked by terrestrial $\mathrm{O}_{2}$ ) were made for several of the stars of Paper I. Most of the spectra had a dispersion of $9 \AA \mathrm{mm}^{-1}$ and were taken with the 3-m telescope of Lick Observatory

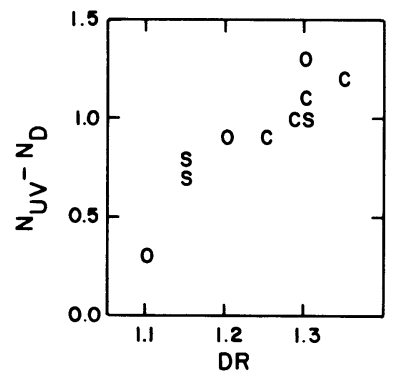

FIG. 2.-Logarithm of the ratio of the column density of $\mathrm{Na} \mathrm{I}$ as deduced from the ultraviolet and from the yellow doublets plotted as a function of the doublet ratio of the yellow Na I lines. Symbols as in fig. 1 with the addition of the O stars, denoted by $\mathrm{O}$. 
TABLE 2

INTERSTELLAR K I OBSERVATIONS

\begin{tabular}{|c|c|c|c|}
\hline Star & $E_{B-V}$ & $W_{\lambda}(\lambda 7699)(\mathrm{m} \AA)$ & $N_{\mathrm{K}}$ \\
\hline \multicolumn{4}{|l|}{ Cloud stars: } \\
\hline HD 147889 & 1.08 & $\leq 50$ & $\leq 11.6$ \\
\hline HD 147701 & 0.72 & $\leq 50$ & $\leq 11.6$ \\
\hline HD 147888 & 0.53 & $\leq 60$ & $\leq 11.7$ \\
\hline$\zeta$ Per $\ldots \ldots \ldots \ldots$ & 0.38 & 100 & 11.9 \\
\hline$\zeta \mathrm{Oph} \ldots \ldots \ldots \ldots$ & 0.32 & 97 & 11.9 \\
\hline \multicolumn{4}{|l|}{ Supergiants: } \\
\hline HD 183143 & 1.30 & 100 & 11.9 \\
\hline HD $192660 \ldots \ldots \ldots$ & 0.88 & 110 & 12.0 \\
\hline
\end{tabular}

and the Varo image tube through the kindness of Dr. R. P. Kraft. One spectrum was kindly obtained by Dr. R. Zappala with the new Varo image tube and the 2.5-m telescope. These spectra are not very high quality, and the region is severely contaminated by many terrestrial lines, so that the measured values of $W_{\lambda}$ are probably accurate to \pm 25 percent. The values we obtained, together with that previously published for $\zeta$ Oph (Herbig 1968), are listed in table 2.

It is immediately apparent that while $\mathrm{K}$ I does not appear to be depleted in the outer parts of dense clouds, it may be depleted in the central regions. If we attempt to analyze the ionization equilibrium of potassium with procedures described in Paper I, we obtain approximately

$$
\begin{aligned}
\frac{N(\mathrm{~K} \mathrm{I})}{N(\mathrm{~K})} & =\frac{n_{e}}{10}, \quad \text { for clouds }, \\
& =\frac{4 n_{e}}{10}, \begin{array}{l}
\text { for supergiants (with similar } \\
\text { formulae for } \mathrm{Na} \mathrm{I}),
\end{array}
\end{aligned}
$$

where we assumed $n_{e}=10^{-3} \mathrm{~cm}^{-3}$ for supergiants $\left(n_{\mathrm{H}}=1 \mathrm{~cm}^{-3}\right)$ and $n_{e}=1 \mathrm{~cm}^{-3}\left(n_{\mathrm{H}}=10^{3} \mathrm{~cm}^{-3}\right)$ in the centers of clouds. We then obtain $N(\mathrm{~K}) / N(\mathrm{H})$ is approximately 5 times solar for the two supergiants, while $N(\mathrm{~K}) / N(\mathrm{H})$ is less than 0.02 of the solar value in the centers of clouds, and about solar in the outer parts.

\section{DISCUSSION}

What can we learn from these new observations? We have deduced $\mathrm{K}$ abundances relative to $\mathrm{H}$ greater than solar for the average of the thin interstellar in the line of sight to the supergiants. This must imply some fault in our simple model, which assumed $n_{e}=$ $10^{-3} \mathrm{~cm}^{-3}$ for the supergiants. Such an error should also show up in $\mathrm{Na}$ I. Using the ultraviolet $\mathrm{Na}$ I observations, we correct the abundance of $\mathrm{Na}$ relative to $\mathrm{H}$ derived from the $\mathrm{D}$-lines in Paper I (i.e., table 7) by the mean value of $N_{\mathrm{UV}}-N_{\mathrm{D}}$ for the supergiants and cloud stars to obtain the results listed in table 3 . Note that the cosmic abundance of $\mathrm{Na}$ relative to $\mathrm{H}$ is approximately $\log \mathrm{Na} / \mathrm{H}=-5.8$. Therefore we have exceeded the cosmic $\mathrm{Na}$ abundance for the case of the supergiants by a factor of 40 (this problem was also apparent in table 7 of Paper I, although not as
TABLE 3

Corrected Average Abundances Relative to Hydrogen

\begin{tabular}{lcc}
\hline \hline & \multicolumn{3}{c}{$\log \mathrm{Na} / \mathrm{H}$ Assumed $n_{e}\left(\mathrm{~cm}^{-3}\right)$} \\
\hline Cloud stars $\left(E_{B-V}>0.9\right) \ldots \ldots$ & -7.1 & 1 \\
Cloud stars $\left(E_{B-V}<0.6\right) \ldots \ldots$ & -5.7 & 0.1 \\
Supergiants $\ldots \ldots \ldots \ldots \ldots$ & -4.2 & $10^{-3}$ \\
\hline
\end{tabular}

severely). The explanation for this phenomenon lies within equation (1) which shows that to obtain $N(\mathrm{~K})$ from the observed quantity $N(\mathrm{~K} \mathrm{I})$, we actually require the average of $n_{e}^{-1}$, rather than $n_{e}$. This will be biased toward the regions where $n_{e}$ is small, so that $1 /\left\langle n_{e}\right\rangle$ is less than $\left\langle 1 \mid n_{e}\right\rangle$. The second relevant factor is that our choice of $n_{e}=10^{-3} \mathrm{~cm}^{-3}$ is probably too low. If we wish to reduce the sodium abundance to its cosmic value, we must adopt $n_{e}=0.04 \mathrm{~cm}^{-3}$, a value in good agreement with that determined from pulsar dispersions (Gunn and Ostriker 1970). We then obtain no $\mathrm{Na}$ depletion in the supergiants, perhaps some depletion of $\mathrm{Na}$ in outer parts of clouds, with $\mathrm{Na}$ depleted by up to a factor of 25 in the central parts. The depletions are not as extreme as those of table 7 of Paper I due to the use of abundances derived from the ultraviolet $\mathrm{Na}$ I lines rather than the D-lines. The calculated overabundance of $K$ relative to $H$ for the supergiants arises in the same way, and when the electron density is raised, the $\mathrm{K}$ abundance becomes solar or slightly less for the normal interstellar medium, solar for the edges of the clouds, with probably some depletion in the centers of clouds.

While the column density or abundance relative to hydrogen of an observed ion can be obtained in a straightforward manner, we see that the corrections for the ionization equilibrium are very model dependent. It should be noted that a large part of the calculated depletion arises from the ionization equilibrium. Table 7 of Paper I combined with the ultraviolet $\mathrm{Na}$ observations indicates $\mathrm{N}_{\mathrm{Uv}}(\mathrm{Na} \mathrm{I}) / N(\mathrm{H})$ is only a factor of 4 less in the centers of clouds than in the supergiants, while $N(\mathrm{Ca} \mathrm{II}) / N(\mathrm{H})$ is down by a factor of 12 in the cloud stars as compared with the supergiants. This point was perhaps not adequately stressed in Paper I.

Jenkins and Savage (1974) claim that $\mathrm{Na}$ is significantly depleted in the normal interstellar medium. They base their claim on White's (1973) values of $n_{e}$ determined from $\mathrm{Ca}$ I/Ca II. If they were correct, then the depletions presented here would be overestimates, and perhaps could be completely eliminated for $\mathrm{Na}$, though not for Ca. However, both Hobbs (1974) and I feel that their value of $\mathrm{Na} / \mathrm{H}$ is too low. Seven of the 10 detections of $\mathrm{Ca} \mathrm{I}$ in White (1973) occur in cloud stars (five stars in the $\zeta$ Oph region), and the mean value of $n_{e}$ for the "normal interstellar clouds" derived from these observations is probably too large because of this. Jenkins and Savage are using a value of $n_{e}$ corresponding to $0.15 \mathrm{~cm}^{-3}$ which is almost certainly too large for anything except clouds which are denser than normal. 


\section{SUMMARY}

I have provided new observations of the ultraviolet $\mathrm{Na}$ I doublet and the $\mathrm{K}$ I interstellar lines for a group of cloud stars and supergiants selected from Paper I. The ultraviolet observations demonstrate that the treatment of the velocity dispersions and the correction for galactic rotation used in the previous work provide results correct to within a factor of 2, although the D-lines in general appear to give $\mathrm{Na}$ abundances a factor of 10 lower than do the ultraviolet lines. Thus, the conclusions on $\mathrm{Ca}$ abundances of Paper I are strengthened by the new observations, while the $\mathrm{Na}$ depletions are reduced if the ultraviolet abundances are correct. The K I observations suggest that $\mathrm{K}$ may be depleted only in the centers of dark clouds. They, together with the new ultraviolet $\mathrm{Na}$ observations, indicate the necessity of adopting a mean value of $n_{e}$ of approximately $0.04 \mathrm{~cm}^{-3}$ in evaluating the ionization equilibrium along the line of sight through a typical cross-section of the interstellar medium. The most important remaining problem is to understand more clearly the implications of the discrepancy between abundances derived from the yellow and ultraviolet $\mathrm{Na}$ I lines.

\section{REFERENCES}

Blanco, V., Demers, S., Douglass, G. G., and Fitzgerald, M. P. 1970, Pub. U.S. Naval Obs., Vol. 21, Ser. 2.

Cohen, J. G. 1973, Ap. J., 186, 149.

Gunn, J. G., and Ostriker, J. P. 1970, Ap. J., 160, 979.

Herbig, G. H. 1968, Zs. f. Ap., 68, 243.

Hobbs, L. M. 1974, preprint.

Jenkins, E. B., and Savage, B. D. 1974, Ap. J., 187, 243.

Morton, D. C. and Hayden-Smith, Wm. 1973, Ap. J. Suppl., 26, 332 .
Münch, G. 1968, Nebulae and Interstellar Matter, ed. B. Middlehurst and L. H. Aller (Chicago: University of Chicago Press), p. 375.

Nachman, P., and Hobbs, C. M. 1973, Ap. J., 182, 481.

Schild, R. E., Peterson, D. E., and Oke, J. B. 1971, Ap.J., 166, 95.

Scholz, M. 1972, Astr. and Ap. Suppl., 7, 469.

Spitzer, L., Jr., and Field, G. B. 1955, Ap.J., 121, 300.

White, R. E. 1973, Ap. J., 183, 81.

JUDITH G. COHEN

Kitt Peak National Observatory, P.O. Box 26732, Tucson, AZ 85726 